\title{
A NO-REFERENCE IMAGE CONTENT METRIC AND ITS APPLICATION TO DENOISING
}

\author{
Xiang Zhu and Peyman Milanfar \\ Electrical Engineering Department \\ University of California at Santa Cruz, CA, 95064
}

\begin{abstract}
A no-reference image metric based on the singular value decomposition of local image gradients is proposed in this paper. This metric provides a quantitative measure of true image content, and reacts reasonably to both blur and random noise, so that it can be used in the automatic selection of parameters for image restoration algorithms, especially for denoising filters. Compared with GCV or SURE based approaches, this metric costs a small amount of computation, and does not require the noise to be Gaussian. Simulated and real data experiments demonstrated that our metric can capture the trend of quality change during the denoising process, and can yield parameters that show excellent visual performance in balancing between denoising and detail preservation.
\end{abstract}

Index Terms - parameter optimization, no-reference metric, sharpness, denoising, singular value decomposition.

\section{INTRODUCTION}

Objective image quality metrics can be divided into two main categories: full-reference and no-reference [1]. Some fullreference metrics, including the classical mean-squared error (MSE) and the structural similarity (SSIM) [1], have been widely used in the image processing field. However, since these metrics basically measure the similarity between the target and reference images instead of real image quality, they can hardly be employed in situations where the reference image is not available. For example, we can not use a fullreference metric to select parameters automatically for image restoration algorithms (e.g. denoising, deblurring and superresolution) in real applications.

In any statistical image restoration algorithm, it can be observed that selecting parameters amounts to a tradeoff between bias and variance in the final estimate. One example is the regularization parameter in Bayesian reconstruction approaches. Generally, the larger the parameter is, the more smooth the image content becomes (small variance), while more useful detail and edges are flattened (larger bias). In other words, an ideal no-reference measure that is useful for the parameter optimization problem should take both noise

This work was supported in part by the US Air Force Grant FA9550-071-0365. and blur on the output image into account. However, most no-reference sharpness metrics, which are designed based on local image gradient [2] or edge width [3], can hardly distinguish image quality decay against high frequency behavior due to noise [4].

Some approaches have been developed for solving the parameter optimization problem. Generalized cross-validation (GCV) based methods have been employed for tuning the regularization parameters for various denoising applications [5]. More recently, the methods based on Stein's unbiased risk estimate (SURE) [6], which aims to access MSE without a reference, were proposed for the denoising problem. One successful example is the Monte-Carlo SURE [7] that can be used for arbitrary denoising algorithms. This method probes the denoising operator with additive noise and manipulates the response signal to estimate MSE. However, these approaches are either limited to a small group of denoising algorithms, or require a large amount of calculation (e.g. Monte-Carlo SURE needs the denoising operation run twice to get the result). Besides, many approaches are also only appropriate when the noise is assumed to be Gaussian (which may not be the case in real applications), and generally require an accurate estimation of the input noise variance as well.

In the article [4], we proposed a sharpness metric $H$ capable of detecting both blur and noise. The limitation of this metric is that it requires a prior estimate of noise variance. In this paper, we propose a new no-reference image content metric. This metric does not require any prior knowledge about the test image or noise. Its value drops monotonically either when image becomes blurred or noisy. In the rest of this paper, we first briefly review the definition and properties of local image gradient matrix and its singular values. Then, we give the definition of the new metric. Several experimental results show the performance of our metric in solving the parameter optimization problem for denoising algorithms. Conclusions are provided in the final section.

\section{SINGULAR VALUES OF THE IMAGE GRADIENT MATRIX}

The metric proposed in [4] is based on the (compact) singular value decomposition (SVD) of local gradient matrix. More 
specifically, considering an image of interest $g(x, y)$, we define the gradient matrix of an $N \times N$ local analysis window $\left(w_{i}\right)$ as:

$$
\mathbf{G}=\left[\begin{array}{cc}
\vdots & \vdots \\
g_{x}(k) & g_{y}(k) \\
\vdots & \vdots
\end{array}\right], \quad k \in w_{i}
$$

where $\left[g_{x}(k), g_{y}(k)\right]$ denote the gradient of the image at point $\left(x_{k}, y_{k}\right)$. The dominant orientation of the local window can be calculated by computing SVD of G [8]:

$$
\mathbf{G}=\mathbf{U S V}^{T}=\mathbf{U}\left[\begin{array}{cc}
s_{1} & 0 \\
0 & s_{2}
\end{array}\right]\left[\begin{array}{ll}
\mathbf{v}_{1} & \mathbf{v}_{2}
\end{array}\right]^{T}
$$

where both $\mathbf{U}$ and $\mathbf{V}$ are orthonormal matrices. The column vector $\mathbf{v}_{1}$ represents the dominant orientation of the local gradient field. Correspondingly, the second singular vector $\mathbf{v}_{2}$ is the direction perpendicular to $\mathbf{v}_{1}$. The singular values $s_{1} \geq s_{2} \geq 0$ represent the energy in the directions $\mathbf{v}_{1}$ and $\mathbf{v}_{2}$, respectively.

In [4] we illustrated that $s_{1}$ can serve as a local sharpness metric when the image is clean. Its value drops if the image becomes more and more blurry. On the other hand, if a clean image patch $\mathrm{g}$ is corrupted by the white (though not necessarily Gaussian) noise $\mathbf{n}$, the gradient matrix of the noisy image patch $\hat{\mathrm{g}}$ would become:

$$
\widehat{\mathbf{G}}=\mathbf{G}+\mathbf{G}_{n}
$$

and on average the corresponding singular values can approximately be written as [4]:

$$
\hat{s}_{i} \approx \sqrt{s_{i}^{2}+\xi N^{2} \sigma^{2}}, \quad i=1,2
$$

where $s_{i}$ denotes the singular values of the latent clean patch $\mathrm{g}, \sigma^{2}$ is the noise variance, and $\xi$ is a constant whose value is determined by the gradient estimation filters. ${ }^{1}$ The above equation tells us that $\hat{s}_{1}$ is determined by both $s_{1}$ and $\sigma^{2}$. Given a fixed $\sigma^{2}$, the value of $\hat{s}_{1}$ drops as $s_{1}$ gets decreased, or say when the image patch $\mathbf{g}$ is more blurry. Unfortunately, $\hat{s}_{1}$ is also monotonically increasing with the noise variance $\sigma^{2}$. In other words, $\hat{s}_{1}$ itself cannot be used directly as a metric in the presence of both blur and noise.

\section{NO-REFERENCE IMAGE CONTENT METRIC}

We define the image content metric $Q$ as:

$$
Q=s_{1} \frac{s_{1}-s_{2}}{s_{1}+s_{2}}
$$

Compared with $s_{1}$, it can be seen that in the definition of $Q$ a normalizing ratio is introduced:

$$
R=\frac{s_{1}-s_{2}}{s_{1}+s_{2}}
$$

\footnotetext{
${ }^{1}$ For example, if we use the filters $[-1 / 2,0,1 / 2]$ and $[-1 / 2,0,1 / 2]^{T}$ to estimate image gradients, the corresponding $\xi=1 / 2$.
}

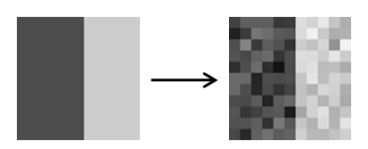

(a)

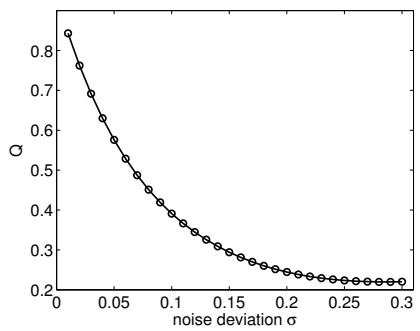

(c)

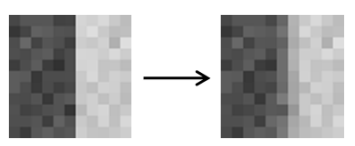

(b)

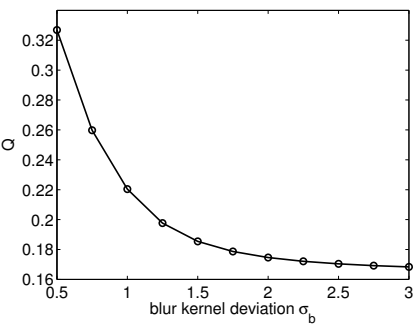

(d)
Fig. 1. Monte-Carlo simulations using both random noise and blur for an edged patch. (a): white Gaussian noise with different variance $\sigma^{2}$ was added to the clean patch. The average $Q$ versus $\sigma$ was plotted in (c). (b): the test patch was blurred first and added by white Gaussian noise with $\sigma=0.1$. The averaged $Q$ was given in (d).

which we call the coherence. Consider a noisy image patch $\hat{\mathrm{g}}$. Its coherence can approximately be calculated by replacing $s_{1}$ and $s_{2}$ with the $\hat{s}_{1}$ and $\hat{s}_{2}$ in (4):

$$
\begin{aligned}
\widehat{R} & \approx \frac{\sqrt{s_{1}^{2}+\xi N^{2} \sigma^{2}}-\sqrt{s_{2}^{2}+\xi N^{2} \sigma^{2}}}{\sqrt{s_{1}^{2}+\xi N^{2} \sigma^{2}}+\sqrt{s_{2}^{2}+\xi N^{2} \sigma^{2}}} \\
& =\frac{s_{1}^{2}-s_{2}^{2}}{s_{1}^{2}+s_{2}^{2}+2 \xi N^{2} \sigma^{2}+2 \sqrt{\left(s_{1}^{2}+\xi N^{2} \sigma^{2}\right)\left(s_{2}^{2}+\xi N^{2} \sigma^{2}\right)}}
\end{aligned}
$$

The above illustrates that in a noisy image patch, the computed value of coherence $R$ is roughly inversely proportional to the local noise variance $\sigma^{2}$ when $s_{1}>s_{2}$, which is true whenever the underlying clean patch contains a strong structure, like an edge. We call these structured patches anisotropic. Under such cases, metric $Q$ serves as an approximation of $\hat{s}_{1} / \sigma^{2}$, which can be thought as an indicator of local signal to noise ratio [4].

As mentioned before, $s_{1}$ and $s_{2}$ represent the energy in both the dominant direction and its perpendicular direction. So basically $R$ measures their relative size. Considering a noise-free region with strong anisotropic geometric structure (such as an edge), the difference between $s_{1}$ and $s_{2}$ is very large, and in the absence of noise, the value of $R$ is near 1 . If white noise is added, the resulting $R$ would be reduced, indicating that this region has become less structured, or the relative strength of the dominant direction has been reduced.

To further understand the performance of metric $Q$ in anisotropic patches in the presence of both noise and blur, we employ Monte-Carlo simulations. In the first experiment, white Gaussian noise with a variety of $\sigma$, ranging from 0.01 to 0.3, was added to a test edged patch (shown in Fig.1 (a)). For each $\sigma, 100$ simulations are carried out with independent noise realizations. Fig.1 (c) plots the averaged $Q$ across 
these realizations, versus $\sigma$. In the second experiment, we take the blurring process into account. The edged patch is blurred by applying a Gaussian smoothing filter with a growing standard deviation $\sigma_{b}$. After that, white Gaussian noise with $\sigma=0.1$ is added respectively. Again, 100 independent noise simulations were applied, and the averaged $Q$ versus $\sigma_{b}$ is given in Fig.1 (d). From this set of experiments, we can see that for anisotropic patches, the value of metric $Q$ drops monotonically as the image content becomes more and more blurred and/or noisy. In other words, only anisotropic patches should be considered when measuring a test image using metric $Q$. Fortunately, these anisotropic patches can be detected by simply thresholding the local coherence $R .^{2}$

\section{APPLICATION TO DENOISING}

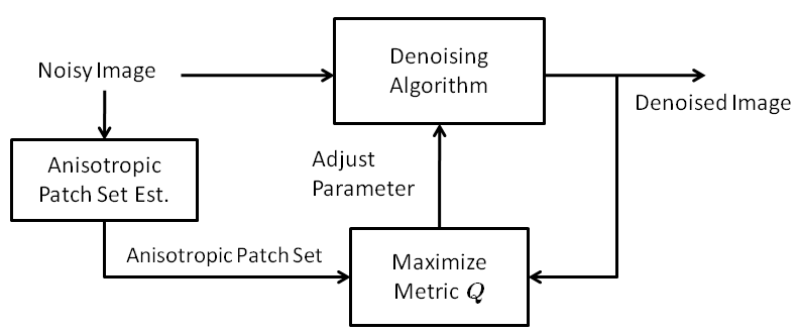

Fig. 2. Selecting the tuning parameter using metric $Q$.

In this section, we will provide evidence showing that the proposed metric $Q$ can be used to optimize the parameters of denoising algorithms. The strategy we take for computing metric $Q$ is as follows:

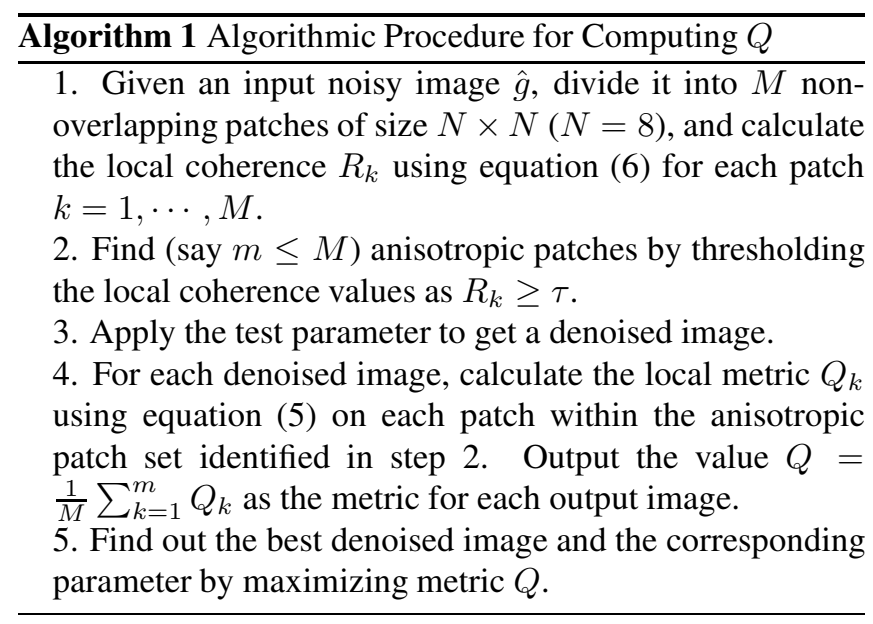

One state of the art denoising algorithm, the blockmatching and 3D filtering (BM3D) [9], is used here as a parameter tuning example. In the BM3D algorithm, a Wiener

\footnotetext{
${ }^{2}$ If $R \geq \tau$, then the patch is detected as anisotropic. This significance test is based on the pdf of $R$ (in noisy isotropic patches). We set the threshold $\tau=0.234$, which is determined by setting the confidence level to be $99.9 \%$. $\tau$ is independent from image content.
}

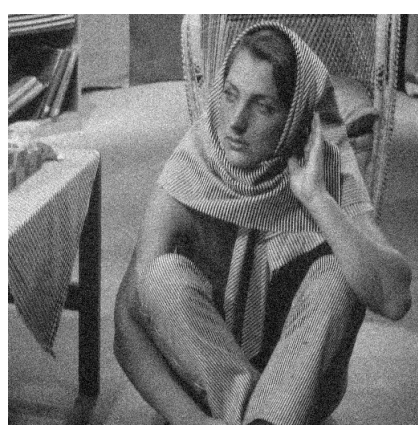

(a) Simulated noisy image

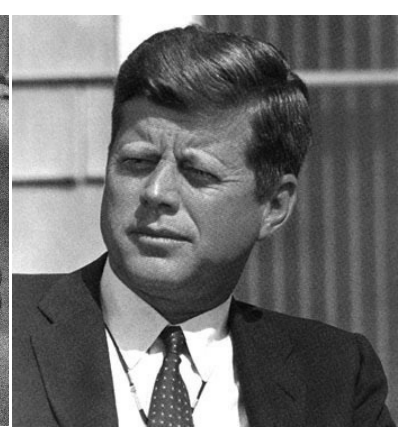

(b) Real noisy image
Fig. 3. Test input images.

filter is employed for collaborative filtering, which requires the estimate of a (variance) parameter $\sigma_{\text {est }}^{2}$. The value of the parameter $\sigma_{\text {est }}$ can strongly affect the output, and thus needs to be tuned. In the following experiments, we optimize this parameter in the broad range of 1 to 30 (the intensity range is $[0,255])$. All the experiments are carried out on a desktop PC with Intel Pentium D CPU $(3.00 \mathrm{GHz})$ in MATLAB. Computing $Q$ for a $512 \times 512$ output image takes around 0.25 second.

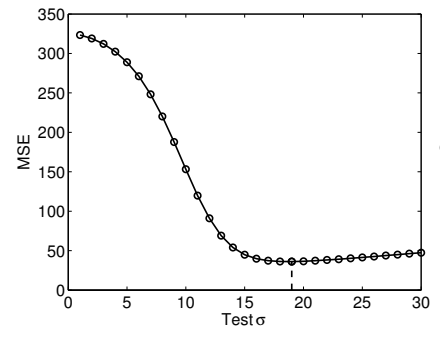

(a) MSE

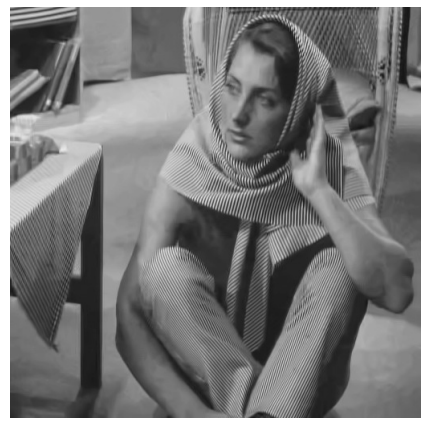

(c) MSE optimized, $\hat{\sigma}_{\text {est }}=19$

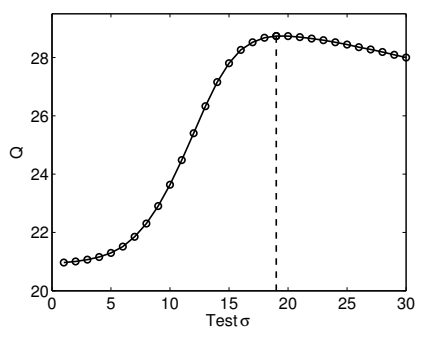

(b) $Q$

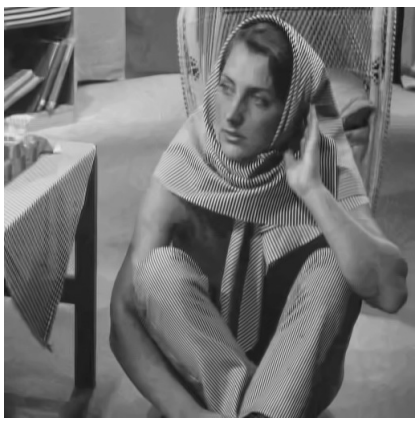

(d) $Q$ optimized, $\hat{\sigma}_{\text {est }}=19$
Fig. 4. Simulated data experiments.

Both simulated and real data experiments are carried out. Due to space limitation, we offer only a small part of the results here ${ }^{3}$. In the simulated experiments, where noisy images were produced by adding white Gaussian noise (see Fig.3 (a)), MSE is also calculated. Plots of the results are given in Fig. 4. It can be seen that metric $Q$ captured the changing trend of quality in the output images. Both $Q$ and MSE

\footnotetext{
${ }^{3}$ The MATLAB code and more experimental results are available at http://users.soe.ucsc.edu/ xzhu/doc/metricq.html.
} 


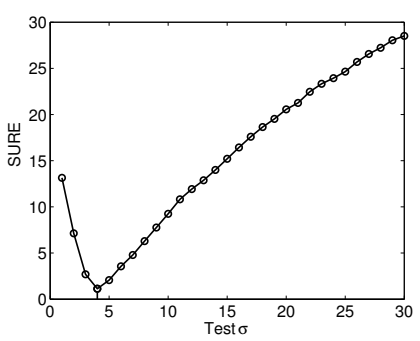

(a) SURE

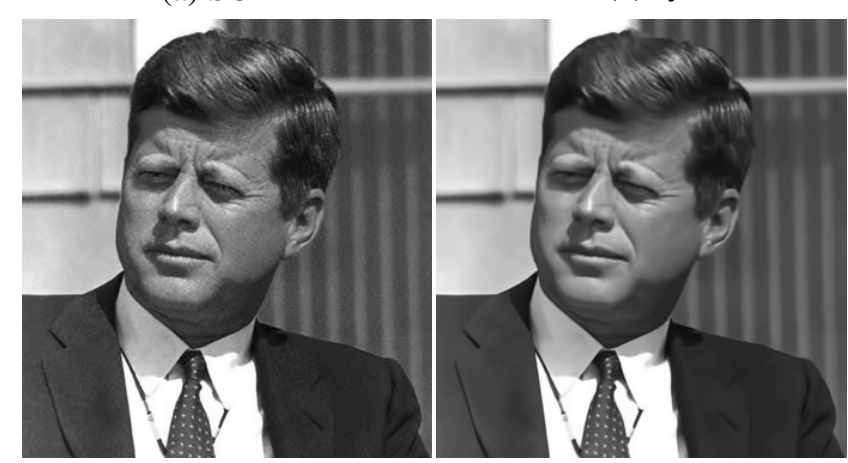

(c) SURE optimized, $\hat{\sigma}_{\text {est }}=4$ (c) $Q$ optimized, $\hat{\sigma}_{\text {est }}=11$

Fig. 5. Real data experiments

show that as the value of the tuning parameter is increased, the overall visual quality of the output image rises first due to the suppression of random noise, and then goes down because of the blurring effect of the filter. The maximum value of $Q$ yielded a very good result (in the given experiment, it offers the same suggestion as MSE) in a completely unsupervised fashion and without access to a reference image.

Fig.3 (b) illustrates a real noisy image, whose noise comes from film grain, scanning and compression processes, and is not Gaussian. Monte-Carlo SURE is implemented as a comparison, where the standard deviation of the probing noise is set to be 0.1 as recommended in [7]. From Fig. 5 we can see that the optimal output image suggested by SURE is inadequate for denoising, as obvious noise can be observed. Meanwhile, the $Q$ optimized image shows good visual performance in balancing between denoising and detail-preservation. This example is not entirely fair to the SURE method since the assumptions (Gaussian noise model) underlying that method are violated in this example. But given the lack of a reasonable alternative, the experiment does illustrate that the metric $Q$ is nevertheless able to maintain its stable performance, indicating that our proposed metric can be useful for a more general variety of practical situations.

\section{CONCLUSION}

In this paper, we proposed an image content metric $Q$ based on the singular value decomposition (SVD) of local image gradients. This metric can reflect the amount of both blur and random noise of images without any prior knowledge, so that it can be used in parameter optimization problems for various image restoration algorithms. We studied the particular case of denoising algorithms here. Simulated and real data experiments demonstrated that this metric can capture the trend of quality change during the denoising process, and can yield parameters that show good visual performance in balancing between denoising and detail preservation.

\section{REFERENCES}

[1] Z. Wang, A. C. Bovik, H. R. Sheikh, and E. P. Simoncelli, "Image quality assessment: From error visibility to structural similarity," IEEE Transactions on Image Processing, vol. 13, no. 4, pp. 600-612, April 2004.

[2] R. Ferzli and L. J. Karam, "A no-reference objective sharpness metric using Riemannian tensor," in Third International Workshop on Video Processing and Quality Metrics for Consumer Electronics, Scottsdale, Arizona, January 2007, pp. 25-26.

[3] P. Marziliano, F. Dufaux, S. Winkler, and T. Ebrahimi, "A no-reference perceptual blur metric," in Proceedings of the International Conference on Image Processing, Rochester, NY, 2002, vol. 3, pp. 57-60.

[4] X. Zhu and P. Milanfar, "A no-reference sharpness metric sensitive to blur and noise," in 1st International Workshop on Quality of Multimedia Experience (QoMEX), San Diego, CA, July 2009, pp. 64-69.

[5] N. Nguyen, P. Milanfar, and G. H. Golub, "Efficient generalized cross-validation with applications to parametric image restoration and resolution enhancement," IEEE Transactions on Image Processing, vol. 10, no. 9, pp. 1299-1308, September 2001.

[6] C. Stein, "Estimation of the mean of a multivariate Normal distribution," Ann. Statist., vol. 9, pp. 1135-1151, 1981.

[7] S. Ramani, T. Blu, and M. Unser, "Monte-Carlo SURE: A black-box optimization of regularization parameters for general denoising algorithms," IEEE Transactions on Image Processing, vol. 17, no. 9, pp. 1540-1554, September 2008.

[8] X. Feng and P. Milanfar, "Multiscale principal components analysis for image local orientation estimation," in Proceedings of the 36th Asilomar Conference on Signals, Systems and Computers, Pacific Grove, CA, November 2002, vol. 1, pp. 478-482.

[9] K. Dabov, A. Foi, V. Katkovnik, and K. Egiazarian, "Image denoising by sparse 3-D transform-domain collaborative filtering," IEEE Transactions on Image Processing, vol. 16, no. 8, pp. 2080-2095, August 2007. 